\title{
EL CAMBIO CLIMÁTICO Y LA RELACIÓN HOMBRE - NATURALEZA
}

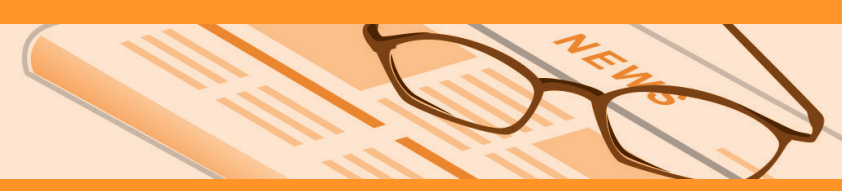

Autor:

Anabel Trujillo Duque ${ }^{1}$

${ }^{1}$ Universidad Tecnológica Equinoccial,

Dirección de Investigación y Transferencia de Tecnologías

Email: atrujillo@ute.edu.ec

Teléf.: 0998108930

Recepción/Received: 2013-09-15 Aceptación/ Accepted: 2013-10-12

Publicado/Published: 2013-12-18

REVISTA DE INVESTIGACIÓN CIENTÍIFICA 


\section{Resumen}

El presente artículo presenta una reflexión alrededor del cambio climático y el calentamiento global, hechos que condicionan el futuro del planeta y de la sociedad en su conjunto. Diversas investigaciones científicas desarrolladas desde los años 70 coinciden en que la intervención del hombre sobre la naturaleza ha alterado de manera permanente el equilibrio del planeta. Basados en la necesidad que tiene la sociedad de alcanzar altos niveles de desarrollo, se ejerce una presión permanente sobre los recursos escasos, lo cual se traduce en una pérdida irreparable de biodiversidad y alteraciones en los ecosistemas. El presente artículo, basado en una extensa revisión bibliográfica, reflexiona sobre el modelo económico que tenemos y el futuro preocupante al que nos enfrentamos como sociedad si no somos capaces de modificar la manera actual de consumo; a la par es un intento por identificar los principales avances y desafíos que plantea para la comunidad internacional la construcción de políticas consensuadas orientadas a prevenir y mitigar los efectos del cambio climático.

Palabras clave: cambio climático, calentamiento global, desarrollo, sociedad de consumo.

\section{Abstract}

The present article offers a reflection about climate change and global warming, facts which make a condition on the future of the planet and the society altogether. Different scientific research works developed since the 70s, coincide that man's mediation over nature has altered the permanent balance of the planet. Based on the necessity that the society has to reach a high level of development, a permanent pressure is applied on the scarce resources, which results in an irretrievable loss of the biodiversity and changes in the ecosystems. This article, which is based in an ample literature review, reflexes on the economic model that we have and the alarming future we will face as a society if we are not able to modify the present way of consumption. Likewise, this paper is an attempt to identify the main advances and challenges that the international community brings up for the construction of reconciled political premises oriented to prevent and soften the climate change effects.

Keywords: climate change, global warming, development, consumer society 


\section{Introducción}

El presente trabajo aborda el calentamiento global, una realidad que condiciona el futuro de todos los seres humanos que habitamos la Tierra y cuya manifestación más evidente es el cambio climático. El modelo económico actual, caracterizado por ser hegemónico y priorizar el crecimiento, amenaza la existencia de recursos críticos para el mantenimiento de la vida, como son el agua, el suelo, los bosques y la biodiversidad; los efectos que tiene la actividad humana sobre una serie de complejos mecanismos que actúan entre sí es lo que provoca los cambios en el planeta. (Duarte, 2006). Con el devenir de los años, el perfeccionamiento de los modelos climáticos ha permitido contar con mayores pruebas que corroboran el cambio acelerado en el clima por efectos de actividades humanas como la agricultura o la tala de bosques.

Es evidente que el modelo actual de acumulación material no favorece a los más pobres ni mejora la calidad de vida de la población mundial; es un modelo excluyente del ser humano y depredador de la naturaleza. Construir un nuevo orden económico, encaminado a priorizar la prosperidad, sin asociarla necesariamente al crecimiento económico y, avanzar en la cimentación de una nueva lógica económica y social, es un reto para los hacedores de políticas y los pensadores de los regímenes internacionales (Jackson, 2009). Sin lugar a dudas, el crecimiento de la economía ayuda a resolver algunos problemas pero crea otros. El consumo excesivo de recursos, expresado en el flujo de materiales y energía necesarios para mantener funcionando la economía provoca una presión sobre complejos mecanismos naturales que ven alterado su funcionamiento, esto provoca los cambios que ahora atraviesa el planeta. En palabras de Randers y Meadows (2006), el crecimiento se ve limitado por la capacidad que tienen las fuentes del planeta para suministrar materiales y energía y de la capacidad de los sumideros para absorber la contaminación y los residuos.

El cambio climático es una amenaza que requiere la formulación de políticas conjuntas entre los países industrializados y los países en desarrollo para transformar la dinámica económica actual. En este escenario es oportuno discutir sobre los regímenes internacionales constituidos para enfrentar el tema y analizar su efectividad en la construcción de acuerdos para enfrentar el cambio climático desde dos aristas: por un lado, la degradación medioambiental y de los recursos naturales, puede ser ocasionada por un excesivo desarrollo económico o, por el contrario, por un desarrollo económico insuficiente, con pocas políticas y mecanismos que permitan reducir los efectos del daño ambiental.

\section{Cambio climático}

Los efectos de la acción humana sobre la Tierra ya no pueden ser ignorados; diversos estudios científicos, como el presentado por el Panel Intergubernamental del Cambio Climático (IPCC), concuerdan en que la intervención del hombre sobre la naturaleza ha alterado de forma permanente los parámetros de equilibrio existentes. En ese punto es importante recordar que la energía que recibe la Tierra desde el Sol debe estar en balance con la radicación emitida desde la superficie terrestre, es decir, debe existir un equilibrio energético. Cualquier factor que genere un cambio sostenido entre la cantidad de energía que entra al sistema (en este caso la Tierra y su atmósfera) y la energía que salen del sistema, pueden generar un cambio climático. Como son factores que nos son partícipes directos del sistema climático, se les conoce como "forzante climático", lo que implica que es un factor que obliga o empuja al clima a un nuevo estado. Las causas de este cambio se dividen en dos categorías generales: causas naturales y causas antrópicas (generadas por actividades humanas). En palabras de Duarte (2006), el cambio climático se refiere a los efectos que la actividad humana tiene sobre el sistema climático global que altera procesos fundamentales del funcionamiento del sistema climático de la Tierra.

El crecimiento exponencial de la población mundial conjuntamente con el industrial ha traído como resultado la alteración del sistema planetario, especialmente por la acumulación de dióxido de carbono en la atmósfera, como consecuencia de la quema de 
combustibles fósiles y la tala de bosques. (Meadows, Randers y Meadows, 2006). Como lo señala el (IPCC, 2007), el calentamiento del sistema climático es inequívoco, como evidencian ya los aumentos observados del promedio mundial de la temperatura del aire y del océano, el deshielo generalizado de nieves y hielo, y el aumento del promedio mundial del nivel del mar. Según Duarte (2006), en los últimos cincuenta años el ser humano ha alterado el funcionamiento y estructura de los ecosistemas de manera rápida y generalizada, sobre todo por fenómenos como la pesca a gran escala, el aumento del uso del suelo para agricultura y sobreexplotación de las fuentes de agua dulce. "Todo indica que de aquí al 2100 el cambio climático ser irá convirtiendo en el principal motor directo de cambio global, determinando cada vez en mayor medida la pérdida de biodiversidad y la alteración del funcionamiento y de los servicios de los ecosistemas terrestres a escala mundial." (Duarte, 2006:93).

La concentración de Gases de Efecto Invernadero (GEI), desde la Revolución Industrial ${ }^{1}$ ha cambiado la temperatura global; es un hecho que los fenómenos climáticos de los últimos años responden a una aceleración de los patrones del clima producidos por efecto de actividades humanas como la agricultura, cambios en el uso del suelo y la quema de combustibles fósiles².

El cuarto Informe del IPCC del año 2007 señala que el calentamiento actual es inequívoco, inusual, antrópico e inquietante, en palabras de Vide (2008: 3 ):

\footnotetext{
1 “Ha existido una fuerte correlación entre las emisiones de $\mathrm{CO} 2$ y el PIB per cápita y, como resultado de ello, desde 1850 , Norteamérica y Europa han producido alrededor del $70 \%$ de todas las emisiones de $\mathrm{CO}_{2}$, mientras que la aportación de los países en desarrollo ha sido inferior al 25\%. La mayor parte de las emisiones futuras procederán de los países hoy día en desarrollo, debido al más rápido crecimiento de su población y de su PIB y a su creciente porcentaje de industrias con uso energético intensivo" Stern Review (2007), La economía del cambio climático, página 14.

2 "Se ha detectado la influencia humana en el calentamiento de la atmósfera y el océano, en alteraciones en el ciclo global del agua, en reducciones de la cantidad de nieve y hielo, en la elevación del nivel medio global del mar y en cambios en algunos fenómenos climáticos extremos. Esta evidencia de la influencia humana es mayor desde el Cuarto Informe de Evaluación. Es sumamente probable que la influencia humana haya sido la causante dominante del calentamiento observado desde mitad del siglo XX." (IPCC; 2013:2).
}

“El cambio climático es Inequívoco, porque las evidencias científicas son suficientes para admitir su realidad. Inusual, o sea, raro, extraño, porque la tasa de aumento de la temperatura es muy alta, con las referencias paleoclimáticas existentes. Antrópico o de origen humano, sin negar, como se ha dicho antes, la variabilidad natural del sistema climático. E inquietante, porque, sin cargar las tintas, los efectos previsibles resultan muy preocupantes"

Existen evidencias científicas que muestran claramente la relación entre la emisión creciente de gases a la atmósfera durante el siglo XX y el incremento de la temperatura global de $0,6^{\circ} \mathrm{C}$ (Duarte, 2006). Como lo señala el Informe del IPCC del año 2007, las emisiones mundiales de GEI por efecto de las actividades humanas se han incrementado, desde la era preindustrial, en un 70\% entre 1970 y 2004. Las actuales concentraciones atmosféricas de $\mathrm{CO}_{2}$, metano $\left(\mathrm{CH}_{4}\right)$, óxido nitroso ( $\mathrm{N}_{2} \mathrm{O}$ ) y halocarbonos exceden los valores naturales registrados en los últimos 650.000 años. "El cambio climático inducido por el hombre está causado por las emisiones de dióxido de carbono ( $\left.\mathrm{CO}_{2}\right)$ y otros gases de efecto invernadero (GEI) que se han acumulado en la atmósfera, sobre todo, durante los últimos doscientos años" (Stern, 2007:17).

La emisión de dióxido de carbono metano y óxido nitroso desestabiliza la temperatura de la Tierra y la gran concentración de GEI aumenta el calor y así se altera el efecto invernadero³ lo que produce el cambio climático.

Como lo señala Duarte (2006), el nivel de consumo de recursos por parte de la humanidad desencadena mecanismos complejos que interactúan entre sí e influyen en los cambios en el planeta que se está constatando actualmente.

${ }^{3}$ El efecto invernadero es un proceso natural que permite a la Tierra permanecer caliente y se produce por la presencia de ciertos gases en la atmósfera. Este fenómeno permite la vida en la tierra, sin él el promedio del clima sería de $23^{\circ}$ bajo cero. En palabras de Duarte (2006), el efecto invernadero es decisivo en el clima del la Tierra y cualquier modificación en dicho mecanismo alteraría el clima. 
Tal y como lo define Costa (2004: 243), "las alteraciones en el balance energético de la Tierra impulsan cambios que van más allá de las variaciones de temperatura, afectan al conjunto de patrones climáticos del planeta"; esto es confirmado con datos del Informe Stern (2007), en donde se indica que las concentraciones de GEI en la atmósfera ha llevado ya a un calentamiento del planeta de más de medio grado Celsius, si el ritmo actual de emisiones no disminuye existe la probabilidad de que la temperatura media global experimente un aumento superior a $2^{\circ} \mathrm{C}$. Sin embargo, la estimación de las variaciones promedio del clima y sus cambios dependerá mucho del modelo de simulación climático utilizado, sin embargo, esto no significa que el cambio climático no se evidencie ya en alteraciones del clima. El cambio climático es el problema más urgente que enfrenta hoy el mundo; de hecho, en el último informe del IPCC, publicado en 2013, se establece que el umbral de 400 ppm de $\mathrm{CO}_{2}$ en la atmósfera fue superado, lo que conlleva al aumento de la temperatura, a un mayor deshielo de las capas polares y a un aumento en el nivel del mar.

Según el IPCC (2007), desde 1990 la Tierra se ha calentado $0,7^{\circ} \mathrm{C}$ y según el ritmo de emisiones actuales tenderá a calentarse más en las siguientes décadas. Esta tendencia desembocará en aquello que el IPCC (2007) denomina “los impactos del cambio climático". Los cambios de temperatura del planeta tendrán un impacto inmediato sobre la disponibilidad de agua y alimentos, la salud y la conservación de los ecosistemas, la erosión del suelo, la degradación de la tierra, así como daños en los arrecifes de coral, en la salinización del agua marina, en la contaminación de los suministros hídricos, en el estrés hídrico por la menor disponibilidad de agua dulce, en el aumento del riesgo de mortalidad por calor, en la escasez de alimentos, en la desnutrición, en el aumento de la temperatura en invierno en los países del hemisferio norte, en el aumento de los costos para la protección costera, en el desplazamiento de poblaciones. Muchos de estos impactos serán irreversibles y afectarán, sobre todo, a los países en desarrollo.

Como lo indica Beck (2007: 21), “[... ] existen buenas razones para predecir que el cambio climático devastará las regiones pobres del planeta, aquéllas donde se solapan el crecimiento demográfico, la pobreza, la contaminación del agua y del aire, las desigualdades entre clases y de género, las epidemias de sida, la corrupción y los gobiernos autoritarios.". El continente más afectado será África, donde la mortalidad por enfermedades como la malaria o el dengue crecerá desproporcionadamente. El mundo se enfrentará a una catástrofe humanitaria sin precedentes. Este punto es coincidente con lo que proponen Cimadamore y Sejenovich (2010), quienes plantean una relación directa entre los desastres climáticos y la pobreza. La variabilidad climática es responsable de la pérdida de cientos de vidas humanas, desplazamientos forzados y crecimiento de la vulnerabilidad social y económica. De acuerdo con Stern (2007: 41-88) "las repercusiones del cambio climático serán cada vez más negativas a medida que las temperaturas crezcan". Más allá de un umbral de $2^{\circ} \mathrm{C}$ está el riesgo de que se produzcan catástrofes ecológicas irreversibles con un alto costo social y económico. El riesgo de no actuar frente al cambio climático tendrá altos costos en el futuro.

Como lo señalan Meadowns y Randers (2006), la Tierra ha llegado a sus límites por efecto del afán de crecimiento económico, el acelerado crecimiento demográfico combinado con el industrial ha alterado profundamente el sistema planetario porque los seres humanos hemos multiplicado el uso de materiales y energía. Esta presión sobre los ecosistemas ha hecho que crezca nuestra huella ecológica4: desde 1980 el mundo ha consumido más recursos de los que el planeta podía regenerar en el mismo periodo. Este comportamiento irracional ha llevado al planeta a sus límites, en muchos casos las fuentes de energía han sido explotadas hasta su agotamiento.

Como lo indica Legget (1996), los problemas del medio ambiente han sido generados por la acumulación. Es innegable que el incremento de los patrones de consumo han modificado el estilo de vida de la población mundial; cada ser humano, industria o Estado debe asumir su responsabilidad en el cambio climático.

${ }^{4}$ La Huella Ecológica es un indicador del impacto ambiental generado por la demanda humana que se hace de los recursos existentes en los ecosistemas del planeta relacionándola con la capacidad ecológica de la Tierra de generar sus recursos. 
La evidencia científica determina que son las acciones humanas las responsables del cambio climático y señala que la única forma de lograr revertir este proceso es modificar el afán de crecimiento económico y consumo sin límites. En este punto es muy importante discutir sobre la disponibilidad y uso de los recursos por parte de los países y sus ciudadanos, "Cualquier conversación sobre la reducción del crecimiento alimenta una amarga disputa sobre el reparto: de los recursos disponibles y de la responsabilidad por el actual estado de cosas. En términos generales, la huella ecológica de un rico es mucho mayor que la de un pobre." (Meadows, Randers y Meadows, 2006: 215). No se trata entonces de limitar el progreso sino de racionalizar el camino para alcanzarlo a través de la implementación de políticas que controlen la extralimitación en la explotación de los recursos.

Diversos autores coinciden en la necesidad urgente de revertir la tendencia actual en el calentamiento global, pues la humanidad está acercándose peligrosamente al umbral que separa lo tolerable de lo catastrófico. Dado que el hombre es el responsable de este proceso, es la humanidad en su conjunto la que debe procurar encontrar los mecanismos capaces de garantizar que los ecosistemas funcionen de manera sostenible. Faross (2000), enfatiza en la necesidad de enfrentar el problema del calentamiento global mediante un planteamiento coordinado entre todos los países para conseguir estabilizar el ritmo de emisión de los GEI. Para este autor, es importante mejorar la eficiencia en el suministro y consumo de energía y buscar alternativas al uso de los combustibles fósiles. Sin embargo, esto no sería suficiente si el modelo capitalista de acumulación no deja de ser la panacea del desarrollo. Es innegable que el estilo de consumo actual no ha ayudado a terminar con fenómenos como el hambre o la pobreza.

\section{Relación hombre - naturaleza}

Para modificar esta tendencia es importante cambiar el paradigma conceptual sobre la naturaleza. Lovelock (2007), en su teoría sobre GAIA señala que es importante imaginar a la Tierra como un ser vivo que opera dentro de ciertos límites y restricciones que han permitido al planeta albergar la vida dentro de una temperatura óptima y de una composición química adecuada. Esta capacidad ha estado presente por más de tres mil millones de años, pero los seres humanos la hemos alterado porque hemos contemplado a la naturaleza como un elemento que debía ser dominado y sometido, no como un elemento más de nuestra vida. Esta visión es coincidente con lo que plantea Boff (1997), quien aboga por la recuperación de la dimensión sagrada de la tierra, lo cual nos permitirá transformar el paradigma imperante en la relación hombre-naturaleza. En lo sagrado está presente aquello que nos llena de entusiasmo, está presente la fascinación por aquello que no entendemos pero que nos conecta con el universo. Para iniciar esta transformación el hombre tiene que cambiar su forma de conectarse con la naturaleza y entender que es parte de ella. La naturaleza no debe entenderse como un conjunto de recursos listos para ser explotados o de una fuerza que debe ser sometida a nuestra voluntad.

Como señala Jiménez (2007), el cambio climático nos da una buena oportunidad para modificar nuestras pautas de desarrollo y buscar caminos alternativos para construir una sociedad más justa económicamente y ambientalmente sostenible. Es importante darnos cuenta que el modelo actual de producción y consumo ha sometido a los ecosistemas a una presión irracional que ya no es sostenible ni permite un desarrollo armónico. Tanto Jiménez, como Boff y Lovelock (2007) se inclinan por un cambio en el paradigma actual de desarrollo, en la necesidad de buscar alternativas que permitan al conjunto de la sociedad la prosperidad pero cuyo fin último no sea la acumulación de riqueza sino la generación de mejores condiciones de vida.

Es innegable que el modelo económico vigente no solo ha contribuido a agrandar la brecha entre ricos y pobres, sino que también ha degradado la naturaleza hasta el punto en el que el desbalance entre la explotación de recursos naturales y el afán de crecimiento económico parece haber llegado al límite, superar este impase demanda de acciones encaminadas a reducir la huella ecológica de la humanidad, para lograrlo existen tres mecanismos: incorporación de tecnología, disminución en el uso de materiales o ampliar la eficiencia. 
La incorporación de estos parámetros es posible y permitiría reducir la huella ecológica actual. La lógica de la economía neoliberal asocia equivocadamente la posesión de bienes materiales con la realización personal, asume que todos los seres humanos deben satisfacer una necesidad creciente de consumo de bienes y servicios, partiendo de la premisa de que "la maximización de la utilidad individual coincide con el bienestar colectivo, dados los beneficios del equilibrio del mercado (óptimo de Pareto)" (Larrea, 2011:26). Como hemos visto, esta lógica depredadora es la que hoy nos ha empujado a una situación crítica.

El cambio climático dejó de ser una excentricidad para convertirse en una realidad. Frente a ello es importante reconocer que "la plenitud humana no se basa en altos niveles de consumo, es importante optimizar la eficiencia social del consumo, minimizando los insumos productivos y los desperdicios requeridos para su producción, para alcanzar la sustentabilidad" (Larrea, 2011: 27).

Como lo señala Daly (1996), el ritmo económico actual ya no es sostenible y es necesario reducir el consumo hasta niveles ecológicamente sustentables y así evitar un colapso ecológico.

Para Jackson (2009), la posesión de bienes materiales no tiene vinculación con la calidad de vida, la convivencia en armonía o con la esperanza. Hasta ahora el paradigma del crecimiento se ha asociado a una mayor prosperidad, sin embargo, esto no se verifica en la realidad. De hecho el crecimiento ilimitado experimentado por el mundo capitalista no ha sido capaz de resolver la desigualdad y la pobreza.

Además el costo ambiental ha sido demasiado alto en términos de la capacidad del planeta para soportar los impactos ambientales producidos por las actividades económicas.

El desafío frente a esta realidad es modificar el paradigma del bienestar como lo hemos conocido hasta hoy y aceptar que el crecimiento ad infinitum en un planeta finito no es posible.

\section{Cambio climático, gobernanza y regímenes internacionales}

A partir de 1987, el tema del cambio climático dejó de ser una preocupación exclusiva de los científicos y empezó a incursionar dentro de las agendas intergubernamentales. La información contenida en el Informe Brundtland (1987), planteo la necesidad de armonizar el desarrollo con el cuidado de la naturaleza y se planteó por primer vez el desarrollo sostenible 5 el cual establece un nivel de responsabilidad con las generaciones futuras por las acciones del presente.

En la Cumbre de la Tierra de Rio de Janeiro desarrollada en 1992 se logró formalizar un marco internacional para la promoción de negociaciones coordinadas por parte de los gobiernos para empezar a controlar aspectos como las emisiones de $\mathrm{CO}_{2}$ y promover prácticas económicas acordes con la sostenibilidad y el respeto por el ambiente. En 1997, por iniciativa de las Naciones Unidas se firmó el Protocolo de Kyoto $^{6}$, cuyo objetivo era reducir las emisiones de seis $\mathrm{GEI}$ que causan el calentamiento global, y cuyo primer periodo de vigencia terminó en el año 2012.

Alrededor de este acuerdo la Unión Europea mantuvo su optimismo y abogó por ampliar sus metas más allá del 2013, pero Estados Unidos siempre buscó negociar otro tipo de protocolo que no afectara su economía.

Las negociaciones alrededor de temas como la deforestación, los bosques o las compensaciones por contaminación se han dilatado y muchos estados no han asumido compromisos firmes para reducir sus emisiones.

${ }^{5}$ El desarrollo sostenible es aquel que satisface las necesidades de las generaciones presentes, sin comprometer la capacidad para que las futuras generaciones puedan satisfacer sus propias necesidades. (Informe Brundtland (1987).

${ }^{6}$ A criterio de autores como Giles (2013), el Protocolo de Kyoto es un ejemplo de la aplicación de un principio de responsabilidad común pero diferenciada, que se evidencia en que sólo algunos Estados asumían el compromiso de reducir la emisión de gases de efecto invernadero al reconocer su mayor responsabilidad en el cambio climático. 
"Las negociaciones para construir un régimen internacional del cambio climático, iniciadas en los años 80, han producido un conjunto de acuerdos (United Nations Framework Conventions on Climate Change (UNFCC), Kyoto, Bonn-Marrakech) nítidamente insuficientes, sujetos a dinámicas que los conducen a un progresivo debilitamiento[... ] Actores como los Estados Unidos, la Federación Rusa y la Unión Europea parecen ser claves en el proceso. De los dos primeros depende que el régimen eche a andar y de la última que no pierda definitivamente el último aliento" (Costa, 2004: 394).

Held y Fane (2009: 19) concuerdan en que las características de muchos de estos tratados internacionales sobre cambio climático son admirables, pero están mal coordinados y su aplicación es débil. Los autores afirman que estos acuerdos “““adolecen de un problema que podemos calificar como "ineficiencia anárquica" en donde se puede identificar gran multiplicidad de actores cuyas labores padecen de una enorme falta de coordinación"”,. Esta ineficiencia anárquica se expresa en aspectos como la representatividad y la responsabilidad.

Si bien en la última década los actores $^{7}$ involucrados en el cambio climático han crecido exponencialmente y a los organismos internacionales se han sumado ONG's, redes de activistas transnacionales, sociedad civil, organismos privados de regulación entre otros múltiples actores, los resultados de sus intervenciones y políticas no han sido capaces de detener el avance de la contaminación o disminuir la emisión de GEI. Esta multiplicidad de voces, como lo señalan Held y Fane (2009), no ha garantizado la eficiencia en el diseño de políticas ambientales a escala global. Al contrario, a menudo ha significado la duplicación de esfuerzos y ha concentrado la representación en Occidente, sobre todo de los países más ricos.

En cuanto a la representación es evidente que el peso político de determinados países ha condicionado el avance en los acuerdos propuestos.

\footnotetext{
7 Los actores más destacados en el tema son: el Sistema de Naciones Unidas representado por la Convención Marco de Cambio Climático, el Grupo de Gestión Ambiental y la Comisión de Desarrollo Sostenible; el Fondo para el Medio Ambiente Mundial; la Directiva Ambiental de la OCDE; el Comité de Comercio y Medio Ambiente de la Organización Mundial del Comercio y; la Cámara del Medio Ambiente de la Corte Internacional de Justicia (Held y Fane; 2009: 119-120).
}

Por ejemplo, en el caso de limitar las emisiones de $\mathrm{CO}_{2}$, la Comunidad Económica Europea ha realizado importantes avances, mientras que países como Estados Unidos ni siquiera han ratificado acuerdos clave como el Protocolo de Kyoto.

A esto hay que añadirle el nivel de responsabilidad de las economías emergentes en la emisión actual de GEI y su corresponsabilidad con el costo ambiental y económico de hacerlo.

En palabras de Leis y Viola (2003), el régimen de cambio climático demanda de la presencia de actores que impulsen, lideren y sustenten los procesos para lograr acuerdos que representen intereses supranacionales y no solo nacionales. En este punto es muy importante que ese actor supranacional sea capaz de cambiar la dinámica de las negociaciones y permita generar acuerdos entre los países desarrollados, emergentes y pobres. En todo caso la implementación o no de los acuerdos depende en gran medida de la voluntad política. En el tema del calentamiento global las iniciativas de un solo país o bloque no son suficientes para que otros las imiten, es importante anotar que la "crisis ecológica global por la gravedad de sus consecuencias ha comprobado la fragilidad de los límites político-territoriales y la irracionalidad de un énfasis exagerado en la soberanía absoluta de los Estados" (Mata, 2008:85). Para Duarte (2006), en cuanto a la gobernabilidad medioambiental, es importante crear marcos y capacidades institucionales que garanticen el acceso equitativo a bienes públicos medioambientales mediante la aplicación de políticas tendientes a solucionar crisis y situaciones de conflicto que puedan presentarse durante la gestión para proteger un recurso.

La consolidación de un régimen internacional sobre cambio climático enfrenta dos retos importantes: lograr que los países industrializados establezcan compromisos en el largo plazo para reducir sus emisiones y conseguir que los países en proceso de industrialización se comprometan a asumir su parte de responsabilidad por la degradación ambiental asociada a sus procesos de desarrollo. Este proceso tiene costos económicos y políticos que hasta ahora han mermado la consolidación de un régimen internacional que 
realmente pueda detener el avance del calentamiento global. Hasta ahora el enfoque bajo el cual se ha analizado el conjunto de acciones para limitar el cambio climático empiezan y terminan en una lógica económica ya sea de incentivos para quienes logran reducir sus emisiones, -o pueden pagar a otros para seguir contaminando-, o fuertes multas para los contaminadores. Las limitaciones que encuentran los procesos de negociación guardan estrecha relación con el hecho de que no se está produciendo un cambio en el paradigma económico. Como lo señalan Cudworth y Hobden (2011), ahora más que nunca es importante repensar al mundo bajo un esquema que represente a lo humano pero también a lo no humano, una agenda en la que se reconfiguren las prioridades para el futuro.

Es claro que la formalización de acuerdos no es suficiente. Enfrentar el calentamiento global es un reto multidimensional que desafía la integración, el orden internacional y plantea la necesidad de generar nuevos mecanismos de gobernanza que superen la fragmentación institucional, la visión particular de un gobierno o de un Estado y que establezca los límites y responsabilidades de los actores involucrados en la toma de decisiones. Como lo señala Innerarity (2012), para conseguir que se haga justicia en el mundo es necesario cambiar el actual orden y buscar que el bienestar de mucha personas no se consiga a costa de la lesión de derechos básicos de millones.

A nivel global esta nueva gobernanza desafía la consolidación de liderazgos fuertes que sean capaces de tender puentes y establecer alianzas efectivas para avanzar sobre temas cruciales como el desarrollo de energías limpias o la ralentización de la economía. A esto se suma la necesidad de ampliar los espacios de discusión y análisis de las políticas mediante una mayor participación de la sociedad civil. En el plano local es importante apuntalar procesos de desarrollo encaminados a disminuir la presión sobre el uso de recursos naturales, desarrollar mecanismos de adaptación y disminución de los riesgos que supone el calentamiento global, así como la necesidad de fortalecer los procesos democráticos en la toma de decisiones. Englobando los desafíos locales como internacionales es claro que el reto más importante es abandonar la visión a corto plazo y empezar a actuar para conseguir mayor inclusión, representatividad y eficiencia en los procesos tendientes a controlar la emisión de GEI.

Las acciones que se tomen tanto a nivel local como global para reducir el impacto del cambio climático deben considerar los costos y beneficios de un proceso que en el presente ya perjudica a los más pobres, pero que el futuro se hará sentir también entre los países desarrollados. Como lo señala el Programa de Naciones Unidas para el Desarrollo (PNUD), en su informe del 2007, es importante encontrar una vía ética y políticamente aceptable que permita avanzar sobre acuerdos con respecto a la distribución de los costos y beneficios de reducir el cambio climático. No podemos permitirnos esperar tener certezas absolutas para actuar frente a este fenómeno, cuando sus consecuencias ya se están sintiendo alrededor del planeta. El cambio climático impacta directamente sobre el desarrollo y está limitando los esfuerzos de la comunidad internacional para reducir la extrema pobreza, sobre todo en aquellos países en donde el aparecimiento de un fenómeno natural catastrófico llega a tener un impacto profundo sobre las condiciones de vida de sus habitantes porque socava las capacidades de comunidades enteras para enfrentar su camino hacia la eliminación de la pobreza y el mejoramiento de sus condiciones de vida.

\section{Conclusiones}

Es evidente que los avances logrados para construir un régimen internacional sobre cambio climático no han logrado articular políticas coherentes para mitigar los efectos inmediatos del calentamiento global. Las acciones encaminadas a reducir las emisiones de GEI han sido insuficientes y alternativas como el desarrollo de nuevas tecnologías para limitar el uso de combustibles fósiles no cuentan con el apoyo económico para hacerlas realidad. Hasta ahora la discusión se ha basado en una lógica economicista centrada sobre quien tiene el derecho a contaminar o quien puede pagar por hacerlo.

El paradigma económico imperante cree todavía que los seres humanos vamos a ser capaces de dominar a la Tierra y someterla a 
nuestra voluntad. Es evidente que ahora ya estamos enfrentando las consecuencias de haber sobreexplotado los recursos naturales y las tragedias ambientales generan altos costos sociales y económicos. En este proceso los pobres son los más afectados porque no cuentan con los recursos para enfrentar las consecuencias del cambio climático. A esto se suma que en las condiciones actuales de desarrollo la redistribución no es una realidad y la gran concentración de riqueza es éticamente inaceptable.

El calentamiento global ya no puede ser ignorado. Es un imperativo desarrollar formas armónicas de convivencia con la naturaleza, equilibrar los niveles de desigualdad y proponer un modelo que no se limite a favorecer la acumulación sino la equidad y la justicia social. El desafío más importante que enfrenta hoy la humanidad es garantizar el sostenimiento de la vida de los seres humanos sobre el planeta. Como nunca antes tenemos ante nosotros un potente motivador para cambiar nuestra conducta y reorientar nuestro accionar. Debemos abandonar la equivocada idea de que estamos aquí para someter a la naturaleza y entender que somos parte de ella y que irremediablemente nuestro destino está asociado al suyo.

Entender que todos los seres humanos somos parte de un mismo y único planeta tal vez nos haga reevaluar nuestras prioridades y concrete definitivamente la atención del mundo sobre la amenaza que significa para la especie humana el calentamiento global. Además es importante reconocer que las acciones desarrolladas por efectos de la industrialización, la quema de combustibles fósiles y el aumento de la frontera agrícola son los responsables de la acumulación de GEI en la atmósfera, no son un fenómeno natural y son una consecuencia asociada al progreso y al crecimiento de la humanidad. Sin duda la opción de no hacer nada no es una posibilidad cuando se trata de enfrentar los desafíos que nos plantea el cambio climático.

Para los regímenes internacionales es un reto superar la visión particular de los estados y construir acuerdos conjuntos que superen la visión económica y de corto plazo.

\section{Referencias Bibliográficas}

Beck, Ulrich (2007). Viviendo en la sociedad del riesgo mundial. Revista CIDOB d'Afers Internacionals, No. 8, pp. 5-57.

Boff, Leonardo (1997). Ecología: grito de la tierra, grito de los pobres. Madrid: Ediciones Trotta, Capítulo 1.

Cimadamore, Alberto., Sejenovich, Héctor (2010). Cambio climático y pobreza. Revista Voces del Fénix No.2, pp. 16-19.

Costa, Oriol (2004), Tesis Doctoral. El estudio de los regímenes internacionales: diagnosis y propuesta, el caso del cambio climático. Universidad Autónoma de Barcelona. Documento consultado el 04/04/2013.

Cudworth, Erika., Hobden, Sephen (2010). Posthuman international relations complesity, ecologism and global politics. Zed Book: New York.

Daly, German (1996). Beyond Growth. Boston: Beacon Press.

Duarte, Carlos (2006). Cambio global, impacto de la actividad humana sobre el sistema Tierra. Consejo Superior de Investigaciones Científicas, Madrid.

Faross, Peter (1990). Cambio climático y contaminación atmosférica: una perspectiva europea, Revista CIDOB d'Afers Internacionals, No. 41, pp. 231-239.

Giles, Rosa (2013). El desafío de la acción internacional en materia de cambio climático después de la reunión de Doha 2012. Boletín Electrónico del Instituto Español de Estudios Estratégicos.

Held, David., Fane, Angus (2009). Democracia, cambio climático y gobernanza global. Revista Papeles de relaciones ecosociales y cambio global, No. 108, pp. 109-130.

Innerarity, Daniel (2012). La gobernanza global, de la soberanía a la responsabilidad. Revista CIDOB d'Afers Internacionals, No. 100, pp. 11-23. 
Jackson, Tim (2009). Prosperidad sin crecimiento, la transición a una economía sustentable. Londres.

Jimenez, Domingo (2007). Cambio climático y cambio de paradigma. Revista Tiempo de paz, No. 85, pp. 16-24.

Larrea, Carlos (2012). Pobreza, desigualdad social y medio ambiente en el Ecuador y América Latina: hacia la definición de una línea de la codicia. Quito: Universidad Andina Simón Bolívar.

Legget, Jeremy (1996). El calentamiento del planeta: informe de Greenpeace, Fondo de Cultura Económica de México, México, 1996.

Leis, Héctor., Viola, Eduardo (2003). Gobernabilidad global posutópica, medio ambiente y cambio climático, Revista Nueva Sociedad No. 185 , pp. 34-49.

Lovelock, James (2007). La venganza de la Tierra, Editorial Planeta Chilena S.A, Santiago de Chile.

Mata Carnevali, María Gabriela (2008). Relaciones Internacionales en clave ambiental, Revista Humania del Sur, Año 3 No. 4, enerojunio, pp.71.92.

Meadows, Donella., Randers, Jorgen., Meadows, Dennis (2006). Los límites del crecimiento 30 años después. Barcelona: Círculo de Lectores, Capítulos 1 y 3.

Panel Intergubernamental de Cambio Climático (2013). Informe sobre Cambio Climático.

Stern, Nicholas (2007). El informe Stern, la verdad del cambio climático, Ediciones Paidós Ibérica S.A, Barcelona.

Vallejo, Beatriz (2010). La responsabilidad de proteger, una nueva dimensión de la soberanía, edición electrónica de la Revista REDALYC, 2011, pp 2 -23.

Vide, Juan. (2008). La nueva realidad del calentamiento global. Un decálogo del cambio climático. Scripta Nova: Revista electrónica de geografía y ciencias sociales, (12), 19. 